\title{
Yapay Zekâ Tabanlı Doğal Dil İşleme Yaklaşımını Kullanarak İnternet Ortamında Yayınlanmış Sahte Haberlerin Tespiti
}

\author{
Mesut Toğaçar ${ }^{1 *(\mathbb{D})}$, Kamil Abdullah Eşidir ${ }^{2}$ (D), Burhan Ergen ${ }^{3}$ (iD \\ ${ }^{1}$ Frrat Üniversitesi, Bilgisayar Teknolojileri Bölümü, Teknik Bilimler Meslek Yüksekokulu, Elazı̆̆, Türkiye \\ ${ }^{2}$ Fırat Kalkınma Ajansı, Elazığ Yatırım Destek Ofisi, Elazı̆̆, Türkiye \\ ${ }^{3}$ Fırat Üniversitesi, Bilgisayar Mühendisliği Bölümü, Mühendislik Fakültesi, Elazığ, Türkiye \\ mtogacar@firat.edu.tr, abdullahesidir@yahoo.com, bergen@ firat.edu.tr
}

$\ddot{O} z$

Sahte haber, bilinçli veya bilinçsiz bir şekilde çeşitli iletişim kanallarını kullanarak yayılan ve hiç bir gerçeklik payı olmayan uydurma haberlerdir. Günümüzde kitleler çoğu haberleri dijital ve sosyal medya üzerinden alıyorlar. Haberlerin hızlı bir şekilde kitlelere aktarabildiği bu tür iletişim ortamlarında çoğu zaman bu haberlerin doğruluğu suiistimal edinilebiliyor. Kökeni bilinmeyen haberler dezenformasyon veya yanlış bilgilendirme yapılarak toplumlarda ciddi sorunlar oluşturabilmektedir. Özellikle internet ortamında bilgi kirliliğine maruz kalan sahte haberler çok hızlı bir şekilde topluma etkisini gösterebilmektedir. Dijital ortamlarda bu tür problemlerin önüne geçilebilmesi için haberlerin doğruluğunu kavrayabilen ve hızlı bir şekilde teyit eden yapay zekâ tabanlı bir yaklaşım bu çalışmada önerilmektedir. Ayrıca, yapay zekânın bir alt dalı olan Doğal Dil İşleme (DDİ) yöntemi ile erişime açık veri setini kullanarak haberlerin gerçek veya sahte olduğunu tespit eden sınıflandırma analizi gerçekleştirildi. Veri seti, 6335 haber başlığ ve içerikten oluşmaktadır. Bu haberlerin 3171'i gerçek haber niteliği taşırken; 3164'ü ise sahte haber niteliği taşımaktadır. Çalışmanın analizinde DDİ yöntemi ile birlikte Uzun Kısa Süreli Bellek (UKSB) modeli kullanıldı ve veri setinin eğitimi bu model sayesinde gerçekleştirildi. Sonuç olarak, bu çalışmada eğitim verilerinden elde edilen genel doğruluk başarısı \% 99,83 idi ve test verilerinden elde edilen genel doğruluk başarısı \% 91,48 idi. Bu sonuçlar bize gösteriyor ki gelecekte düşünmeyi planladığımız benzeri çalışmalara umut verici olmuştur.

Anahtar kelimeler: Doğal Dil İşleme, Yapay Zekâ, Sahte Haber, Derin Öğrenme, Haber Sınıflandırma.

\section{Detection of Fake News Published on the Internet Using Artificial Intelligence}

\section{Based Natural Language Processing Approach}

\begin{abstract}
Fake news is fabricated news that spread consciously or unconsciously through various communication channels and has no real share. Today, the masses receive most news on digital and social media. In such communication environments, where news can be transferred to the masses quickly, the accuracy of this news can often be abused. News of unknown origin can cause serious problems in societies by making disinformation or misinformation. Especially, fake news exposed to information pollution in the internet environment can show its effect on society very quickly. To prevent such problems in digital environments, an artificial intelligence-based approach that can grasp the accuracy of the news and confirm it quickly is proposed in this study. In addition, a classification analysis was performed using the Natural Language Processing (NLP) method, a sub-branch of artificial intelligence, to determine whether the news was real or false using the dataset that was accessible. The dataset consisted of 6335 news headlines and content. While 3171 of this news is real news; 3164 is fake news. In the analysis of the study, the Long Short Term Memory (LSTM) model was used together with the NLP method and the training of the dataset was carried out with this model. As a result, the overall accuracy success from the training data was $99.83 \%$, and the overall accuracy success from the test data was $91.48 \%$. These results show us that similar studies that we plan to think about in the future have been promising.
\end{abstract}

Keywords: Natural Language Processing, Artificial Intelligence, Fake News, Deep Learning, News Classification.

\footnotetext{
* Sorumlu yazar.

E-posta adresi: mtogacar@firat.edu.tr
} 


\section{Giriş (Introduction)}

Geçmiş zamandan günümüze yanlış bilgiler ve sahte haberler çeşitli amaçları gerçekleştirme doğrultusunda kullanılmış ve süreç içerisinde tartışmalara yol açmıştır. Günümüzde ise bu durum internet ortamında daha hızlı bir şekilde hedef kitlelere ulaşmaktadır ve etkisi de kısa bir sürede görülmektedir. Zaman içerisinde anlık gelişen bilgilerin kontrolü zorlaşmaktadır ve internet ortamlarında paylaşılan bilgi ve haberler çoğu zaman kaynağı belirtilmeden yayınlanmaktadır. Bu durum sosyal medya üzerinden (Whatshapp, Facebook, Twitter vb.) kitlelere hızlı bir şekilde ulaşmaktadır ve kitleleri hızlı bir şekilde etkileşime geçirebilmektedir (Ünal ve Taylan, 2017). Haber kaynaklarının doğruluğunun teyit edilmesi ve yayınlanmadan önce belirli bir olgunluk seviyesine gelmesi gerektiği habercilik anlamında etik ve ahlaki bir kuraldır. Bu kural bazen kişiler tarafından bir an önce haberleştirme duygusu, hırs, çıkar ilişkisi, vb. sebepler ön plana çıktığı zaman bozabilmektedir (Figdor, 2017).

Bilgilerin ve haber içeriklerinin hızlı bir şekilde dezenformasyon olduğu bir internet ortamında bu durumu kontrol etmek zorunluluk haline gelmiştir. Küresel dünyada bu sürecin insanlar tarafindan kontrolü zor ve imkânsızdır (Jayaseelan vd., 2020). Bu durumda insanların kontrol mekanizmasını makinelere aktaran bir sisteme ihtiyaç duyulmuştur ve adını yapay zekâ denilen kavram gelişmiştir. Yapay zekâ teknolojisi, insandaki idrak edebilme, düşünebilme, karar verme gibi mantıksal ve duyusal yetenekleri taklit ederek makineler tarafından bu eylemlerin gerçekleştirilmesini sağlar (Sun vd., 2020). Bu süreçte donanımları kontrol edebilen çeşitli yazılım dilleri (MATLAB, Python, R Studio vb.) geliştirilmiştir ve bu yazılımlar üzerinde yapay zekânın alt bir dalı olan derin öğrenme modelleri (AlexNet, GoogLeNet, ResNet, LSTM vb.) tasarlanmıştır (Doğan ve Türkoğlu, 2019). Milyarlarca kullanıcının anlık bulunduğu internet ortamında sahte haberlerin önüne geçebilmek için yapay zekâ tabanlı sistemlerin kullanılması zaruri bir ihtiyaç haline gelmiştir. $\mathrm{Bu}$ çalışmada hedefimiz, yapay zekâ teknolojisinin alt dalı olan Doğal Dil İşleme (DDİ) yöntemini kullanarak sahte haberlerin tespitini gerçekleştirmektir. Son zamanlarda yapay zekâ tabanlı sahte haber tespitini gerçekleştirmede birçok makaleler yayınlanmıştır. $\mathrm{Bu}$ makalelerden bazıları incelenirse; (Kaliyar vd., 2020) çalışmasında, sahte haber türlerinin sinıflandırılmasında FNDNet adını verdikleri bir derin öğrenme modeli geliştirdiler. Onlar, FNDNet modeli ile birlikte Hiper Parametre optimizasyon yöntemini de kullandılar ve elde ettikleri sınıflandırma başarısı \% 98,36'di. (Altunbey Özbay ve Alataş, 2020), sahte haber verilerinin tespiti için çeşitli yapay zekâ modellerini kullandılar. Onlar, çalışmasında kullandıkları veri setini farklı oranlarda ayırarak modeller tarafindan eğitimi gerçekleştirmişlerdir ve elde ettikleri sinıflandırma başarısı \% 97,4'di. (S ve Chitturi, 2020) çalışmasında, sahte haber verilerinin tespiti için DDİ yaklaşımı ile birlikte UKSB modelini kullandılar. Onların bu çalışmadan elde ettikleri sınıflandırma başarısı \% 91, 32 'di. (Horne ve Adali, 2017) çalışmasında, iki sınıflı haber verisini kullanmışlardır. Onlar çalışmasında Kelime Hesap yöntemini kullanarak analizleri gerçekleştirdiler ve elde ettikleri sınıflandırma başarısı \%71'di. (Onan, 2021) çalışmasında, kitlesel çevrimiçi kurslardan bireylerin duygu sinıflandırılmasını topluluk öğrenme ve derin öğrenme yaklaşımlarını kullanarak gerçekleştirmiştir. En iyi başarıyı UKSB ağları ve GloVe tekniğini birlikte kullanarak elde etmiştir. Duygu siniflandırma sürecinde $\% 95,80$ oranında genel doğruluk başarısı sağlamıştır. (Yüksel ve Tan, 2018) çalışmasında, sosyal ă̆ veri tabanını kullanarak insanların görüşlerini gerçek zamanlı olarak yorumlayabilen akıllı karar destek sistemi önermişlerdir. Onlar çalışmasında özellik seçimi, gereksiz kelime çıkarımı gibi ön işlemleri el ile gerçekleştirmişlerdir. Duygu analizi için Google ara yüz uygulamasını kullanmışlar ve önerdikleri yaklaşımı İngilizce ve Türkçe kelimeler için ayrı ayrı uygulamışlar. Onlar çalışmasında Türkçe için \%84,49 ve İngilizce için \%95 sınıflandırma doğruluğu elde etmişler.

Bu makalenin bölüm özeti şu şekildedir; veri seti ile ilgili bilgiler ikinci bölümde yer almıştır. Derin öğrenme modelleri ve kullanılan yöntemler ile ilgili açıklamalar üçüncü bölümde yer almıştr. Deneysel analizler ve sonuçları dördüncü bölümde; tartı̧̧ma ve sonuç bölümleri ise sırasıyla son iki bölümde yer almıştır.

\section{Veri Seti (Dataset)}

Veri seti dört sütun özellikten oluşmuş erişime açık "csv" uzantılı bir dosyadır. Sütun özelliklerinde; haber kimlik numarası, haber başlığı, haber içeriği ve haber durumu (sahte, gerçek) yer almaktadır. Haber durumu sütunu iki sınıflı bir haber içeriği olduğunu bize vermektedir. Haberler 2016-2018 yılları arasında uluslararası medyada gündem bulmuş internet verilerini içermektedir ve yazım dili İngilizce olarak yer almaktadır. Veri seti, Romanya Akademisi Biyokimya Enstitüsü'nde görev yapan araştırmacı Raluca Chitic tarafından Kaggle web sitesinde erişime açılmıştır (Chitic, 2021). 3171 adet gerçek haber içeriğinden ve 3164 adet sahte haber içeriğinden oluşmuştur. Toplamda 6335 adet haber başlığ ve içerikten oluşmuştur. Bu durum ile ilgili çubuk grafiğgi Şekil 1'de gösterildi. Veri setinin sütun özelliklerini ve örnek içeriklerini gösteren tasarım Şekil 2'de gösterildi. Bu çalışmanın deneysel analizinde veri setinin $\% 25$ 'i test verisi ve $\% 75$ 'i eğitim verisi olarak ayrıldı. 


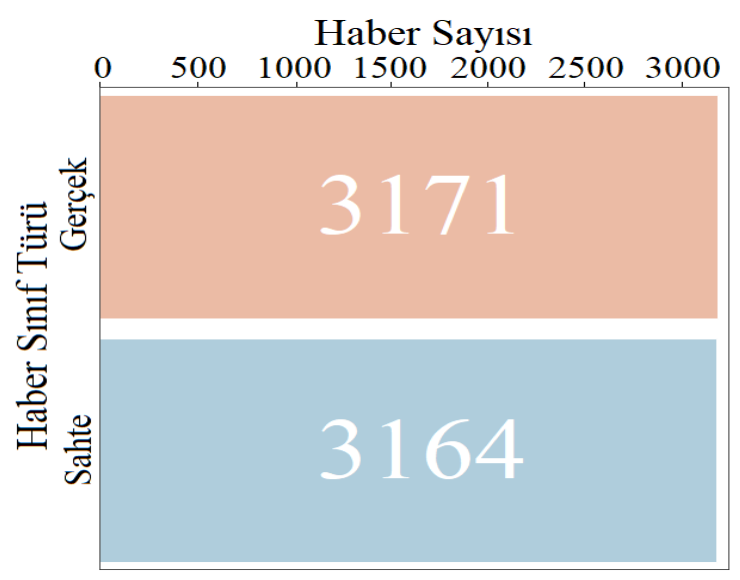

Şekil 1. Veri setinin istatistik bilgilerini gösteren çubuk grafiği (Bar graph showing statistics information of the dataset)

\begin{tabular}{|c|c|c|c|}
\hline Haber No & Haber Başlık & Haber İçerik & Sinif \\
\hline 3608 & Kerry to go to Paris in gesture of sympathy & U.S. Secretary of State John F. Kerry said Mon... & REAL \\
\hline 875 & The Battle of New York: Why This Primary Matters & It's primary day in New York and front-runners... & REAL \\
\hline 95 & 'Britain's Schindler' Dies at 106 A & A Czech stockbroker who saved more than $650 \mathrm{Je} . .$. & REAL \\
\hline 4869 & Fact check: Trump and Clinton at the 'commande... & Hillary Clinton and Donald Trump made some ina... & REAL \\
\hline 2909 & Iran reportedly makes new push for uranium con... & Iranian negotiators reportedly have made a las... & REAL \\
\hline 8476 & You Can Smell Hillary's Fear & Daniel Greenfield, a Shillman Journalism Fello.. & FAKE \\
\hline 10294 & Watch The Exact Moment Paul Ryan Committed Pol & Google Pinterest Digg Linkedin Reddit Stumbleu.. & FAKE \\
\hline 10142 & Bernie supporters on Twitter erupt in anger ag... & Kaydee King (@KaydeeKing) November 9, 2016 T.. & FAKE \\
\hline 6903 & Tehran, USA & Inl'm not an immigrant, but my grandparents .. & FAKE \\
\hline 7341 & Girl Horrified At What She Watches Boyfriend D... & Share This Baylee Luciani (left), Screenshot o.. & FAKE \\
\hline
\end{tabular}

Şekil 2. Veri setinin haber sınıflarını gösteren örnek içerik şeması (Sample content diagram showing the news classes of the dataset)

\section{Derin Öğrenme Modeli, Yöntemler (Deep Learning Model, Methods)}

\subsection{Uzun Kısa Süreli Bellek Modeli (Long Short Term Memory Model)}

UKSB modeli tekrarlayan sinir ağ 1 (TKA) modelinden türetilmiş bir modeldir. TKA modelinin yapısında döngüler vardır ve bu döngüler sayesinde geri bildirimler önceki ve sonraki katmanlar arasında olunabilir. Böylece kısa bilgiler TKA modeli tarafindan öğrenilebilir. Ancak uzun süreli bilgiler TKA modeli için bir sorundur ve çözüm için UKSB modeli tasarlanmıştır. UKSB modeli TKA modelinin aksine tutulması gereken bilgiler tek bir sinir ağı kullanmak yerine özel ve çok katmanlı sinir ağları kullanarak doğrusal etkileşimle çalışır. Girdi verileri doğrusal zincir boyunca kolayca katmanlar arasında geçiş sağlar. UKSB modeli katmanlar arasında bilgi geçişlerini özel kapılar ile gerçekleştirir. UKSB modelinde eğer bir bilgi ağ yapısında kullanılacaksa "giriş kapıları" ile bilgilerin aktarımı sağlanır. Eğer bilgiler gereksizce veya hafızada yeterli bir alan oluşturulacak ise "unutma kapıları" kullanılır. UKSB tarafından "unut ve giriş" kapıları birleştirilecekse bu durumda "güncelleme kapıları" kullanılır. Neticede, UKSB modeli TKA modeli gibi sadece görüntüleri kullanarak bilgi aktarımı gerçekleştirmez; video, ses gibi verilerde de bu işlemi başarılı bir şekilde gerçekleştirir ve işlenen verilerde "çıkış kapılarına" aktararak tahmin, sınıflandırma adımlarını gerçekleştirir (Le vd., 2019). UKSB modelinin işlem adımlarını gösteren tasarım Şekil 3’te gösterildi.

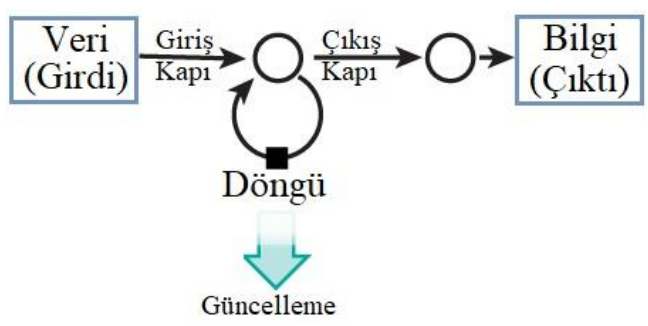

Şekil 3. UKSB modelinin işlem adımlarını gösteren tasarım (Design showing the process steps of the LSTM model)

$\mathrm{Bu}$ çalışmada kullanılan UKSB modelinin katmanları ile ilgili temel bilgiler Tablo 1'de verildi. 
Veri analizi için kullanılan metinsel ifadeler UKSB modelinin Girdi katmanında işlenir. LSTM katmanları ile işlenen veriler yoğun bir şekilde elde edilen özellikler Yoğunluk katmanına girdi olarak aktarılır. Yoğunluk katmanı ise kendi içerisinde aktivasyon fonksiyonlarını kullanarak (Softmax, ReLU, Sigmoid) (Wang vd., 2020) çıkış parametresini düşürtür ve haber verisinin sınıflandırma olasılığını çıktı olarak aktarır. Biz bu çalışmada üç aktivasyon fomksiyonunu sırasıyla Yoğunluk katmanlarında kullandık. Modelin eğitiminde optimizasyon algoritması olarak Adam (Bock ve Weiß, 2019) yöntemi tercih edildi ve öğrenme oranı ise $10^{-5}$ seçildi.

Tablo 1. UKSB modelinin bu çalıșmada tercih edilen katman ve parametre bilgileri (Preferred layer and parameter information of the LSTM model in this study)

\begin{tabular}{lcr}
\hline Katman & Çıkış Şekli & $\begin{array}{r}\text { Parametre } \\
\text { Sayıs1 }\end{array}$ \\
\hline Embedding (Girdi) & $(300,100)$ & $1,000,000$ \\
LSTM (UKSB) & $(300,128)$ & 117,248 \\
LSTM (UKSB) & 64 & 49,408 \\
Dense (Yoğunluk) & 32 & 2,080 \\
Dense (Yoğunluk) & 16 & 528 \\
Dense (Yoğunluk) & 1 & 17 \\
\hline
\end{tabular}

\subsection{Doğal Dil İşleme Yöntemi (Natural Language Processing)}

DDİ, sosyal medya internet ortamındaki verilerin anlamlı ve istenen bilgilerin dijital cihazlar tarafindan öğrenilmesini sağlayan yapay zekâ destekli bir yöntemdir. DDİ yöntemi akıllı uygulamalarda (sohbet botları, dil çeviri ortamları, bilgi özetleme, spam tespiti, intihal yazılımları vb. ) kullanılmaktadır (Ong vd., 2020). DDİ yöntemi ön işlem adımları olarak; kelime normalizasyonu, gürültü azaltma ve nesne standartı oluşturma tekniklerini kullanır. Kelime normalizasyon tekniğinde, aynı kelime kökünden gelen (örneğin; gidiyorum, gidecek, gittim vb.) sözcükleri normalizasyonunu gerçekleştirir. Gürültü azaltma tekniğinde; bağlaç sözcüklerini (ve, veya, ile vb.) cümleler içerisinde tespitini edebilmek ve anlam karmaşasını ortadan kaldırmaktadır. Nesne standart oluşturma tekniğinde ise; toplumsal veya dijital ortamlarda kullanılan kısaltmaların (örneğin; RT: retweet) anlamlarını tespit eden bir tekniktir. DDİ yöntemi ön işlem tekniklerinin ardından, kelimeleri oluşturan cümlelerin özne-nesne-yüklem ilişkisini tespit eden varlık çıkarma sürecini gerçekleştirir. Bunun dışında cümlelerin içerisinde geçen kelimelerin frekans bilgileri, sayısı gibi bilgiler de DDİ yöntemi ile tespit edilebilmektedir (Zhou vd., 2020). DDİ yönteminin işlem adımları Şekil 4’te gösterildi.

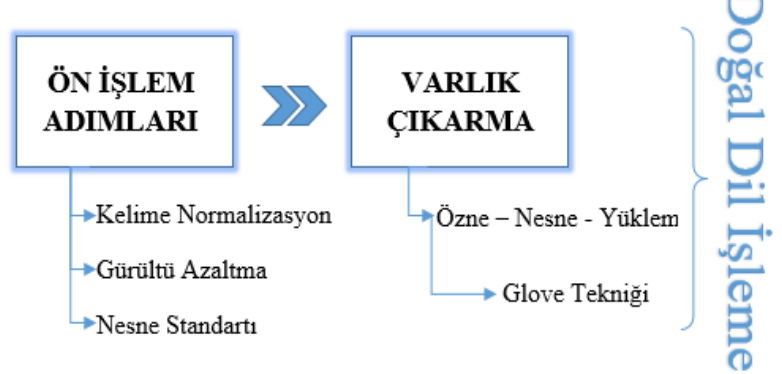

Şekil 4. Doğal dil işleme yönteminin adımları (Steps of the natural language processing method)

DDİ yönteminin adımları arasında kelime normalizasyonu, ilk adımı oluşturmaktadır. Bu adım "okudum, okuyorum, okuyacağım" gibi aynı kökten türeyen kelimelerin normalize oluşumu üzerinde işlemler gerçekleştirir. Gürültü giderme adımı genel olarak cümlede kullanılan bağlaçlar (ve, veya, ya da, ama, vb.) üzerine işlemlerin yürütülmesi ile alakalıdır. $\mathrm{Bu}$ tür bağlaçlar cümle içerisinde gereksiz kullanılmaktadır. Bunun tespitinde cümlelerin genlik değeri ile bağlaçların genlik değerleri arasında bir oran belirlenir. Bu oran sayesinde gürültü genliğinin artması engellenir (Adalı E, 2016). Nesne standart adımı herkes tarafından kabul edilmiş kısaltmaların ( $\mathrm{dm}$ : direct message, rt: retweet, vs.) üzerinde yapılması öngörülen bir tekniktir. Bu üç adımın verilere uygulanması ile "ön işleme" süreci tamamlanmış olur ve "Varlık Çıkarma" adımına geçiş sağlanır. "Varlık Çıkarma" adımında ilk uygulanacak işlem; cümlenin özne, nesne ve yüklem kelimelerinin belirlenmesi ve ayırt edilmesidir. Varlık Çıkarma tekniğini gerçekleştiren, Python dilinde tasarlanmış açık kaynak kodlu kütüphaneler mevcuttur. Bu çalışma için "nltk" kütüphanesinin kod parametreleri kullanıldı. Son adımda "Glove" tekniği kullanılarak verilerin istatistiksel bilgileri, kelime sayısı, yoğunluğu, kelime bulut gösterimi gibi bilgilerin elde edilmesi sağlandı. Kısacası "Glove" tekniği olasılık istatistiklerinden yararlanarak veri setinin istatistiksel bilgilerini vermeye yarar. $\mathrm{Bu}$ işlemin gerçekleşmesinde Eşitlik (1)'de ifade edilen matematiksel formül kullanır. $\mathrm{Bu}$ eşitlikte, kelimelerin birlikte bulunma sayımlarının matris değeri X ile temsil edilmektedir. $X_{i j}$ değişkeni, $i$. kelime bağlamında $j$. kelimenin kaç defa geçtiğini tablo halinde gösterimini sağlar. $V$ değişkeni, kelime dağarcığının boyutunu temsil eder. Son olarak denklemde kullanılan $b$ değişkeni, ön ayar değerini temsil ederken; $W$ değişkeni ise kelime vektörlerini temsil eder (Pennington, Socher, ve Manning, 2015).

$$
J=\sum_{i, j=1}^{V} f\left(X_{i j}\right)\left(W_{i}^{T} W_{J}+b_{i}+b_{j}-\log X_{i j}\right)^{2}
$$

Bu çalışmada DDİ yöntemi olarak Python yazılım dili kullanıldı ve "nltk" kütüphanesi kullanıldı ve varlık çıkarma işlemlerin gerçekleştirilmesinde "Glove" tekniği kullanıldı. Glove tekniği, kelimelerin vektörel 
olarak gruplanmasını sağlayan ve vektör temsillerini elde edebilmek için denetimsiz bir öğrenme gerçekleştirmektedir (Pennington, Socher, ve Manning, 2015). Ayrıca, bu çalışmada kullanılmış DDİ yöntemi ile ilgili açık kaynak kodlar Python dilinde tasarlandı (Madz, 2021).

\section{Deneysel Analiz (Experimental Analysis)}

Çalışmanın deneysel analizleri Google Colab üzerinden gerçekleştirilmişstir ve kullanılan yazılım dili Python 3.6'd1. Python kodlarının tasarlanması ve derlenmesi Jupyter Notebook ara yüzü kullanılarak gerçekleştirildi. Deneysel analizler için karmaşıklık matrisi kullanıldı. Karmaşıklık matrisinin hesaplanmasında kullanılan metrikler ise; Duyarlılık (Duy), Özgüllük (Özg), Hassasiyet (Has), F-skoru (Fskr) ve Doğruluk (Dğr)'dir. $\mathrm{Bu}$ metriklerin hesaplanmasında Eşitlik (2) ile Eşitlik (6) arasındaki formüller kullanıldı. Eşitliklerde kullanılan değişkenler ise; Doğru Pozitif (DP), Doğru Negatif (DN), Yanlış Pozitif (YP), Yanlış Negatif (YN)'dir (Demir 2021; Sertkaya, Ergen, ve Togacar, 2019).

$$
\begin{aligned}
& \text { Duy }=\frac{D P}{D P+Y N} \\
& \text { Özg }=\frac{D N}{D N+Y P} \\
& \text { Has }=\frac{D P}{D P+Y P} \\
& F-s k r=\frac{2 \times D P}{2 \times D P+Y P+Y N} \\
& \text { Dğr }=\frac{D P+D N}{D P+D N+Y P+Y N}
\end{aligned}
$$

Deneysel analizde kullanılan veri setinin \%25'i test verisi olarak ayrıldı. Derin öğrenme modeli için tercih edilen mini-batch parametresinin değeri 128 seçildi. Mini-batch, eğitimin gerçekleştirildiği donanımsal özellikler ile doğrudan bağlantılıdır ve bu parametre ile modelin eğitim esnasında aynı anda ne kadar veri işleneceğini bize verir (Yang vd., 2019). Deneyin eğitiminde tercih edilen devir sayısı 100 seçildi. Eğitim verilerinin analizinde elde edilen genel doğruluk başarısı $\% 99,83$ 'di ve test verilerinden elde edilen genel doğruluk oranı ise \%91,48'di. Eğitim-test başarısını gösteren grafik Şekil 5'te gösterildi. Eğitim-test kaybını gösteren grafik ise Şekil 6'da gösterildi. Bu çalışmanın test verilerinin diğer analiz sonuçları ise; Duyarlılık başarısı \%92,76'di, Özgüllük başarısı \%90,25' di, Hassasiyet başarısı \%90,09 ve F-skor başarısı \%91,41'di. Analiz hesaplamaların gerçekleştirildiği karmaşıklık matrisi Şekil 7'de gösterildi ve analiz sonuçları ise Tablo 2' de verildi.

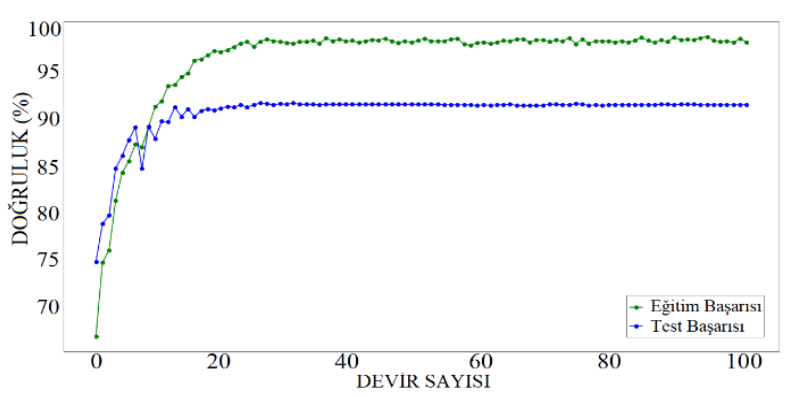

Şekil 5. Bu çalışmanın eğitim-test verilerine ait başarı grafikleri (The success graphs of the training-test data of this study)

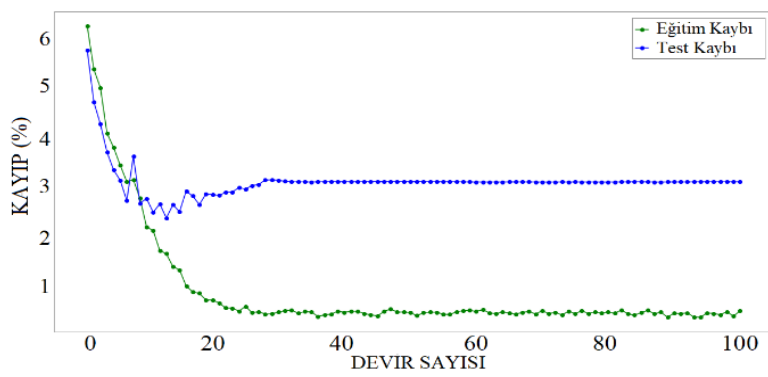

Şekil 6. Bu çalışmanın eğitim-test verilerine ait kayıp grafikleri (The loss graphs of the training-test data of this study)

Tablo 2. Deneyin karmaşıklık matrisinden elde edilen analiz sonuçları (Analysis results obtained from the confusion matrix of the experiment)

\begin{tabular}{cccccccc}
\hline Model ve Yöntem & Sinıf & $\begin{array}{l}\text { F-Skr. } \\
(\%)\end{array}$ & $\begin{array}{l}\text { Duy. } \\
(\%)\end{array}$ & $\begin{array}{c}\text { Özg. } \\
(\%)\end{array}$ & $\begin{array}{c}\text { Has. } \\
(\%)\end{array}$ & $\begin{array}{c}\text { Dğr. } \\
(\%)\end{array}$ & $\begin{array}{c}\text { Genel } \\
\text { Dğr. }(\%)\end{array}$ \\
\hline $\begin{array}{c}\text { UKSB } \\
\& \\
\text { DDİ } \\
\& \\
\text { Glove }\end{array}$ & Sahte & 91,41 & 90,09 & 92,88 & 92,76 & 91,48 & 91,48 \\
\hline
\end{tabular}




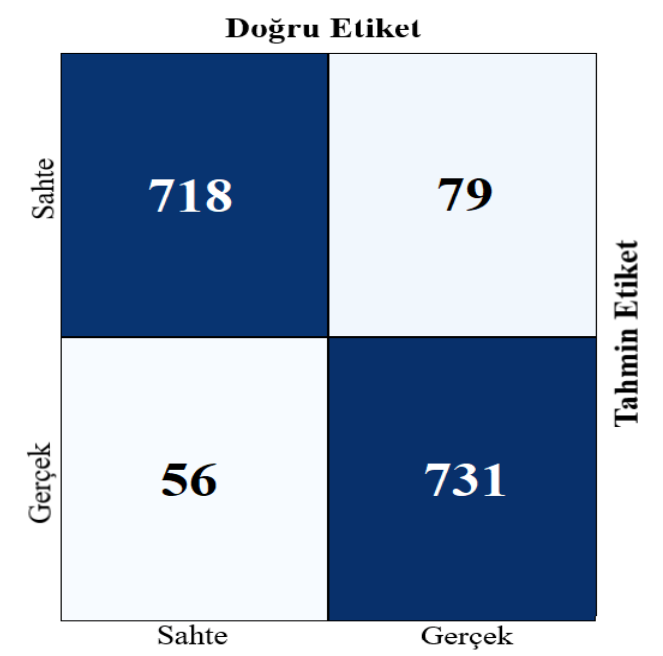

Şekil 7. Deneysel analiz sonucu elde edilen karmaşıklık matris grafiği (Confusion matrix graph obtained as a result of experimental analysis)

Deneyin son aşamasında sahte ve gerçek haberlerin kelime başlıklarının yer aldığı kelime bulut görseli oluşturuldu. Buradaki amacımız sahte haberlerin etiket görselleri ile gerçek haberin etiket görselini karşılaştırmaktı. Kelime bulutlarının olușturulmasında python yazilımında yer alan "Word Cloud" parametresi kullanıldı. Kelime bulutlarının sağladığ yarar, istenilen anahtar kelimelerin okuyucuya görselleştirilerek sunulmasıdır. Böylece daha çok okur tarafından ilgi çekilmesi amaçlanmaktadır (Atenstaedt, 2012). Sahte haberlerin kelime bulut şeması Şekil 8'de gösterildi ve gerçek haberlerin kelime bulut şeması Şekil 9'da gösterildi. Şekil 8 ve Şekil 9 karşılaştırıldığında okuyucu gözüne doğrudan etkileyen büyük etiketler birbirinden farklıdır. Bu durum haberlerin sahte ve gerçek olma durumları ile doğrudan ilgilidir.

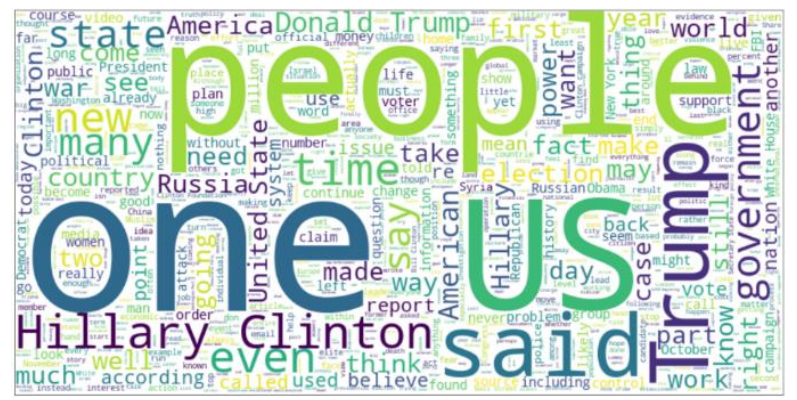

Şekil 8. Sahte haberlerin kelime bulut gösterimi (Word cloud representation of fake news)

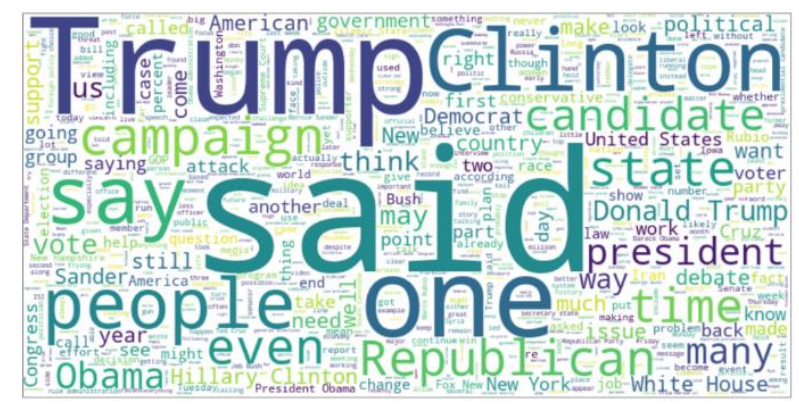

Şekil 9. Gerçek haberlerin kelime bulut gösterimi (Word cloud representation of real news)

\section{Tartışma (Discussion)}

Sahte web haberlerinin bilinçli veya bilinçsiz bir şekilde sayısının son zamanlarda artması toplumlarda kaosa kadar sürüklenebilecek bir problem haline gelmiştir. Bu tür durumlarda insanlar tarafindan önlem alınması milyarlarca kitlesi olan sosyal ve dijital medyada önüne geçilemez bir vaziyettir. Çünkü saniyesinde kontrol edilmeyen haber hedefindeki kitlelere hızlı bir şekilde yayılabilmektedir. Peki, bu durumun önüne nasıl geçilir veya nasıl minimize edilir? Aslında problemin çözümü problemin oluştuğu kaynağın kendisindedir. Yani yine çözüm sosyal ve dijital medyay 1 kontrol edebilen milyarlarca bilgiyi anlık bir zamanda süzebilen yazılımlar ile çözülebilir. Bizde bu yaklaşıma uyarak çalışmamızda yapay zekâ tabanlı UKSB ve DDİ yöntemlerini modelleyerek sahte haberleri algılayabilen bir yaklaşım sunduk. Çalışmamıza benzer model ile katkı sunan bir çalışmada (S ve Chitturi, 2020) tarafindan 2018 yilında gerçekleştirmiştir. Bu çalışma ile ilgili analiz sonucu Tablo 3'te verildi.

Tablo 3. Benzer yaklaşım ile gerçekleştirilmiş çalışmaların karşılaştırılması (Comparison of studies carried out with a similar approach)

\begin{tabular}{ccccc}
\hline Makale & Y11 & $\begin{array}{c}\text { Test } \\
\text { Verisi } \\
(\%)\end{array}$ & $\begin{array}{c}\text { Model } \\
/ \\
\text { Yöntem }\end{array}$ & $\begin{array}{c}\text { Dğr. } \\
(\%)\end{array}$ \\
\hline $\begin{array}{c}\text { ( Sreekumar } \\
\text { ve Chitturi) } \\
\text { Bu çalışma }\end{array}$ & 2020 & 20 & UKSB, DDİ & 91,32 \\
\hline
\end{tabular}

(Sreekumar ve Chitturi, 2020) çalışmasında "George McIntires Fake News" açık erişimli veri setini (McIntire, 2017) kullandılar. Onlar, UKSB ve DDİ yöntemlerini modelleyerek sahte haberlerin tespitini $\% 91,32$ oranında bir başarı ile gerçekleştirdiler. Onların kelimeler üzerinde özellikler çıkartarak bu başarıyı elde ettiler. Ayrıca çalışmalarında test verisi oranı onların $\% 20$ 'di. Bizim çalışmamızda ise bu oran $\% 25$ 'di. Bizim eğitim veri oranımız onların eğitim verisi oranına göre daha düşüktür; fakat daha iyi performans elde edilmiştir. $\mathrm{Bu}$ sınıflandırma farklılığ parametreler ve değerleri ile alakalı olduğunu düşünüyorum. Ayrıca onların kullandığı veri seti sayısı 
bizim kullandığımız veri setinden düşüktü. Sonuçta iki çalışmada benzer modeller ile farklı veri seti türünden yakın doğrulama sonucu almışlardır.

Çalışmamızın test başarısını artırmak için farklı optimizasyon yöntemlerini de (SGD, Adadelta vb.) analiz ettik; fakat Adam yöntemi ile elde edilen başarının üzerinde bir sonuç elde edemediğimiz için diğer optimizasyon yöntemlerinin sonuçlarını analizlere eklemedik. Bunun dışında UKSB modelinin yoğunluk katman sayısını artırmayı denedik; fakat bu denememizde başarıyı artırmadığı için en ideal model katmanları ile sınıflandırmayı gerçekleştirdik.

\section{Sonuçlar (Conclusions)}

Yakın zamana kadar toplumlar görsel ve basılı medya aracılığı ile haberleri takip edebilmekteydiler. Fakat son zamanlarda bu durum sosyal ve dijital medya üzerinden yürütülmektedir. Haberlerin aktarıldığı bu tip iletişim ortamlarında hızlı bir şekilde bilgiler yayıldığ için etkisi de büyük olabilmektedir. Bu makale ile sosyal ve dijital medya ortamlarında sahte haberlerin önüne geçebilmek için yapay zekâ tabanlı bir analiz gerçekleştirilmiştir. Çalışmanın amacı sahte haberlerin tespitinde ileri teknolojik yaklaşımları bir arada kullanarak başarılı bir şekilde gerçekleştirilmesini sağlamaktı. Bizde, UKSB ile beraber DDİ yöntemini modelleyerek haber içerikli veri setinin analizini gerçekleştirdik. Eğitim verilerinde elde edilen doğruluk başarısı \%99,83'di ve test verilerinden elde edilen doğruluk başarısı \%91,48'di. Bu sonuçlar bize sosyal platformlar üzerinde gerçekleştireceğimiz çalışmalara umut verici oldu.

Gelecek çalışmada, Türkçe haber web sitelerinden oluşturulmuş veri setleri üzerinden sahte haberlerin ve yanlış bilgilerin tespitini farklı yaklaşımlar ile birlikte analizleri gerçekleştirilecektir.

\section{Kaynaklar (References)}

Adal1, E., 2016, "Doğal Dil İşleme”. Türkiye Bilişim Vakfi Bilgisayar Bilimleri ve Mühendisliği Dergisi , 5 (2). https://dergipark.org.tr/tr/pub/tbbmd/issue/22245/238797

Altunbey Özbay, F., ve Alataş B, 2020, "Çevrimiçi Sosyal Medyada Sahte Haber Tespiti.”, DÜMF Mühendislik $\begin{array}{llll}\text { Dergisi, } & 11 & \text { (1): }\end{array}$ https://doi.org/10.24012/dumf.629368.

Atenstaedt, R., 2012, "Word Cloud Analysis of the BJGP.", British Journal of General Practice, 62 (596): 148 LP 148, https://doi.org/10.3399/bjgp12X630142.

Bock, S., ve Weiß M., 2019, “A Proof of Local Convergence for the Adam Optimizer.", In 2019 International Joint Conference on Neural Networks (IJCNN), 1-8, https://doi.org/10.1109/ijenn.2019.8852239.

Chitic, R., 2021, "REAL ve FAKE News Dataset." Kaggle, 2021, https://www.kaggle.com/rchitic17/real-or-fake.

Demir, F., 2021, "DeepCoroNet: A Deep LSTM Approach for Automated Detection of COVID-19 Cases from Chest XRay Images.”, Applied Soft Computing, 103: 107160. https://doi.org/https://doi.org/10.1016/j.asoc.2021.107160

Doğan, F., ve Türkoğlu İ., 2019, “Derin Öğrenme Modelleri ve Uygulama Alanlarına İlişskin Bir Derleme", DÜMF $\begin{array}{llll}\text { Mühendislik } \quad \text { Dergisi } 10 & \text { (2): 409-45, }\end{array}$ https://doi.org/10.24012/dumf.411130.

Figdor, C., 2017. "(When) Is Science Reporting Ethical? The Case for Recognizing Shared Epistemic Responsibility in Science Journalism.”, Frontiers in Communication, 2: 3, https://doi.org/10.3389/fcomm.2017.00003.

Horne, Benjamin D., ve Adali S., 2017, “This Just In: Fake News Packs a Lot in Title, Uses Simpler, Repetitive Content in Text Body, More Similar to Satire than Real News.", http://arxiv.org/abs/1703.09398.

Kaliyar, Rohit K., Anurag G., Pratik N., ve Soumendu S., 2020, "FNDNet - A Deep Convolutional Neural Network for Fake News Detection.”, Cognitive Systems Research, 61: $\quad 32-44$. https://doi.org/https://doi.org/10.1016/j.cogsys.2019.12.0 05 .

Le, Xuan H., Hung Viet H., Giha L., ve Sungho J., 2019, "Application of Long Short-Term Memory (LSTM) Neural Network for Flood Forecasting.", Water (Switzerlve), 11 (7). https://doi.org/10.3390/w11071387.

Madz, 2021, "NLP Using GloVe Embeddings.” Kaggle, 2021, https://www.kaggle.com/madz2000/nlp-using-gloveembeddings-99-8-accuracy.

McIntire, G., 2017, "Machine Learning Finds 'Fake News' with $88 \%$ Accuracy." KD Nuggets, 2017, https://www.kdnuggets.com/2017/04/machine-learningfake-news-accuracy.html.

Ong, Charlene J., Agni O., Rebecca Z., Francois Pierre M. C., Meghan H., Liang M., Darian F., vd., 2020, "Machine Learning ve Natural Language Processing Methods to Identify Ischemic Stroke, Acuity ve Location from Radiology Reports.”, PLOS ONE, 15 (6): e0234908. https://doi.org/10.1371/journal.pone.0234908.

Onan A., 2021, "Sentiment analysis on massive open online course evaluations: A text mining and deep learning approach.”, Comput. Appl. Eng. Educ, 29: 572-589. doi:10.1002/cae.22253.

Pennington, J., Socher R., ve Manning C.D., 2015, "GloVe: Global Vectors for Word Representation." Stanford University, 2015, https://nlp.stanford.edu/projects/glove/.

Jayaseelan R., Brindha D., ve Kades W, 2020, "Social Media Reigned by Information or Misinformation About COVID-19: A Phenomenological Study.", SSRN Electronic Journal, https://doi.org/10.2139/ssrn.3596058.

Sreekumar D., ve Chitturi B., 2020, "Deep Neural Approach to Fake-News Identification.", Procedia Computer Science, 167: 2236-43, https://doi.org/https://doi.org/10.1016/j.procs.2020.03.27 6.

Sertkaya, M. E., Ergen B., ve Togacar M., 2019, "Diagnosis of Eye Retinal Diseases Based on Convolutional Neural Networks Using Optical Coherence Images.”, In 2019 23rd International Conference Electronics, 1-5. https://doi.org/10.1109/electronics.2019.8765579.

Sun, Shaojing, Yujia Zhai, Bin Shen, ve Yibei Chen. 2020. "Newspaper Coverage of Artificial Intelligence: A Perspective of Emerging Technologies." Telematics ve 
Informatics,

101433.

https://doi.org/https://doi.org/10.1016/j.tele.2020.101433.

Ünal, R., ve Taylan A., 2017, "Sağlık İletişiminde Yalan Haber - Yanlış Enformasyon Sorunu ve Doğrulama Platformları.", Atatürk İletişim Dergisi / Dergi Park. https://dergipark.org.tr/tr/pub/atauniiletisim/issue/34005/ 360148.

Wang, Y., Li Y., Yong S., ve Rong X., 2020, “The Influence of the Activation Function in a Convolution Neural Network Model of Facial Expression Recognition.”, Applied Sciences (Switzerlve), $10 \quad$ (5). https://doi.org/10.3390/app10051897.

Yang, Z., Wang C., Zhang Z., ve Li J., 2019, “Mini-Batch Algorithms with Online Step Size." Knowledge-Based $\begin{array}{lll}\text { Systems, } & \text { 165: 228-40. }\end{array}$ https://doi.org/10.1016/j.knosys.2018.11.031.

Yüksel A.S., Tan F.G., 2018, "A real-time social networkbased knowledge discovery system for decision making", Automatika.,

59: 261-273. https://doi.org/10.1080/00051144.2018.1531214.

Zhou, M., Nan D., Shujie L., ve Heung-Yeung S., 2020, "Progress in Neural NLP: Modeling, Learning, ve Reasoning.", Engineering, 6 (3): 275-90. https://doi.org/10.1016/j.eng.2019.12.014. 